\title{
Construction Management at risk in der deutschen Bau- und Immobilienwirtschaft
}

\author{
Empirische Untersuchung der kritischen Erfolgsfaktoren
}

\author{
Bertram Laibach $\mathbb{D}$
}

Eingegangen: 3. Februar 2018 / Angenommen: 15. März 2019 / Online publiziert: 2. April 2019

(C) Der/die Autor(en) 2019

Zusammenfassung In Reaktion auf die häufigen Misserfolge bei der Projektentwicklung bemüht sich die deutsche Bau- und Immobilienwirtschaft sowie die öffentliche Hand seit längerer Zeit, partnerschaftlich ausgerichtete Projektorganisationsformen in Form von Wertschöpfungspartnerschaften zu etablieren, die an das amerikanische Construction Management at risk (CM at risk) angelehnt sind. Während diese Form der Immobilienbeschaffung im angloamerikanischen Raum zur Reduzierung von Ineffizienzen beigetragen hat und die Vorteilhaftigkeit durch empirische Analysen belegt ist, wird das Effizienzsteigerungspotenzial dieser Beschaffungsvariante in Deutschland bislang sehr kritisch beurteilt. Die Erfahrungen der Projektbeteiligten mit CM at risk fallen in Abhängigkeit der individuellen Ausgestaltung der Projektstruktur sehr unterschiedlich aus.

In einer empirischen Studie wurden die in Deutschland praktizierten Gestaltungsformen von $\mathrm{CM}$ at risk hinsichtlich ihres Organisationsaufbaus, des Verfahrensablaufs sowie der eingesetzten Instrumente zur Gestaltung und Steuerung untersucht. Die Analyse zeigt neben der starken Heterogenität der Abwicklungsmodelle, dass Organisationsaufbau und Verfahrensablauf häufig in einem deutlichen Gegensatz zur partnerschaftlichen Grundphilosophie der Wertschöpfungspartnerschaft stehen und zum Teil ungeeignete Steuerungs- und Vergütungsmechanismen eingesetzt werden. Die starke Heterogenität in der organisatorischen und vertraglichen Ausgestaltung von $\mathrm{CM}$ at risk führt zwangsläufig zu sehr unterschiedlichen Ergebnissen in Bezug auf die Effizienz und damit den Erfolg der Wertschöpfungspartnerschaft.

Die Studie legt die komplexen Kausalzusammenhänge zwischen der institutionellen Ausgestaltung von CM at risk und dem Verhalten der ökonomischen Akteure während der Projektabwicklung offen und identifiziert die kritischen Erfolgsfaktoren zur Gestaltung und Steuerung einer effizienten Wertschöpfungspartnerschaft.

B. Laibach $(\bowtie)$

RKS Ingenieure GmbH, Saaläckerstraße 8, 63801 Kleinostheim, Deutschland

E-Mail: blaibach@rks.de 
Dieser Beitrag liefert erstmals ein umfassendes Bild über die organisatorische und vertragliche Ausgestaltung, die Problemfelder in der Praxis sowie die kritischen Erfolgsfaktoren von CM at risk in der deutschen Bau- und Immobilienwirtschaft. Die Ergebnisse der Untersuchung sind die Voraussetzung für die Entwicklung eines theoretischen Modells und leisten somit einen innovativen Beitrag zur Standardisierung und erfolgreichen Etablierung von $\mathrm{CM}$ at risk in Deutschland.

Schlüsselwörter Immobilienprojektentwicklung · Projektorganisation ·

Organisationsmodell · Partnering · Entscheidungsunterstützung ·

Wertschöpfungspartnerschaften

\title{
Construction management at risk in the German construction and real estate industry
}

Empirical study of critical success factors

\begin{abstract}
In reaction to the frequent failures in property development, the German construction and real estate industry as well as the public sector try to establish partnership-oriented project delivery systems in the form of value-added partnerships that are based on the American construction management at risk (CM at risk). While this form of real estate procurement in the Anglo-American region has contributed to the reduction of inefficiencies and the advantages have been substantiated by empirical analyzes, the efficiency enhancement potential of this procurement method in Germany has so far been assessed very critically. The experiences of the project participants with $\mathrm{CM}$ at risk are very different depending on the individual design of the project structure.

In an empirical study, the multiple variants of $\mathrm{CM}$ at risk practiced in Germany were examined regarding their organizational structure, the procedure and the instruments used for design and control. In addition to the strong heterogeneity of the procurement models, the analysis shows that organizational structure and procedure are often in contrast to the partnership philosophy of the value-added partnership and that partly inappropriate control and remuneration mechanisms are used. The strong heterogeneity in the organizational and contractual structure of CM at risk inevitably leads to very different results in terms of efficiency and thus the success of the value-added-partnership.

The study exposes the complex interactions between the institutional design of $\mathrm{CM}$ at risk and the behavior of the economic actors during project execution and identifies the critical success factors for implementing and managing an efficient value-added-partnership.

For the first time, this article provides a comprehensive picture of the organizational and contractual structure, the problem areas in practice and the critical factors of success of CM at risk in German construction and real estate industry. The results of the study are the precondition for the development of a theoretical organization model and are an innovative contribution to the standardization and successful establishment of CM at risk in Germany.
\end{abstract}


Keywords Property development - Project organization - Organization model · Partnering $\cdot$ Decision support · Value-added-partnerships

\section{CM at risk als innovative Projektorganisationsform}

Jeder Bauherr muss zu Beginn eines Projektes die richtungsweisende Entscheidung treffen, in welcher Konstellation Planung, Ausführung und Management des Projektes realisiert werden sollen. Er muss hierbei unter Berücksichtigung verschiedener Rahmenbedingungen diejenige Projektabwicklungsform für sein Projekt auswählen, die eine möglichst optimale Verfolgung der Bauherrnziele gewährleistet.

Die in Deutschland praktizierten konventionellen Projektorganisationsformen, Einzelgewerkevergabe und Generalunternehmer- bzw. Totalunternehmervergabe, weisen erhebliche strukturelle Defizite auf, so dass es bei Immobilienprojekten immer wieder zu massiven Termin- und Kostenüberschreitungen sowie erheblichen Qualitätsmängeln kommt. Die vielfältigen Probleme resultieren hierbei u.a. aus dem Umstand, dass die konventionellen Projektorganisationsformen den veränderten Anforderungen an die Projektabwicklung und damit die Projektorganisation in zunehmendem Maße nicht mehr gerecht werden.

In den vergangenen Jahrzehnten wurden daher vermehrt Wertschöpfungspartnerschaften als alternative Formen der Projektorganisation wie z. B. Öffentlich-Private Partnerschaften (ÖPP), Partnerschaftsmodelle bzw. Partneringkonzepte oder GMPModelle (vgl. Gralla 2001; Tautschnig/Hulka 2002; Mathoi 2006; Pfnür 2011) diskutiert und in einigen Projekten mit stark unterschiedlichem Erfolg umgesetzt. Diese basieren jeweils auf CM at risk, das als eigenständige Projektorganisationsform in den USA bereits sehr lange und erfolgreich praktiziert wird (vgl. Mütze et al. 2009, S. 119 und Ohler et al. 2012, Rdn 191).

$\mathrm{CM}$ at risk basiert im Gegensatz zu konventionellen Projektorganisationsformen auf dem Managementansatz des Partnering sowie der frühzeitigen Integration von Realisierungs-Know-How in der Planungsphase. Die Erfahrungen speziell in den USA zeigen ein erhebliches Effizienzsteigerungspotenzial gegenüber konventionellen Projektorganisationsformen durch Kosteneinsparungen, Bauzeitreduzierungen sowie Qualitätssteigerungen (vgl. Konchar 1997). Darüber hinaus lässt sich eine grundlegende Verbesserung des Verhältnisses zwischen den Projektbeteiligten einhergehend mit einem Rückgang gerichtlicher Auseinandersetzungen feststellen (vgl. Schlabach 2013, S. 187 und Bennett und Jayes 1995, S. 20). Die Untersuchung von zwei Pilotprojekten in Deutschland zeigt ebenfalls, dass immobilienwirtschaftliche Wertschöpfungspartnerschaften die Effizienz der Projektentwicklung erheblich verbessern können (Meyer und Pfnür 2015, S. 59).

\section{Verfahrensablauf und Organisationsstruktur von CM at risk}

$\mathrm{CM}$ at risk gliedert sich grundsätzlich in eine Preconstruction Phase und eine Construction Phase. Nachdem der Construction Manager (CM) vom Auftraggeber (AG) im Rahmen eines Kompetenzwettbewerbs ausgewählt wurde, erbringt dieser in der 


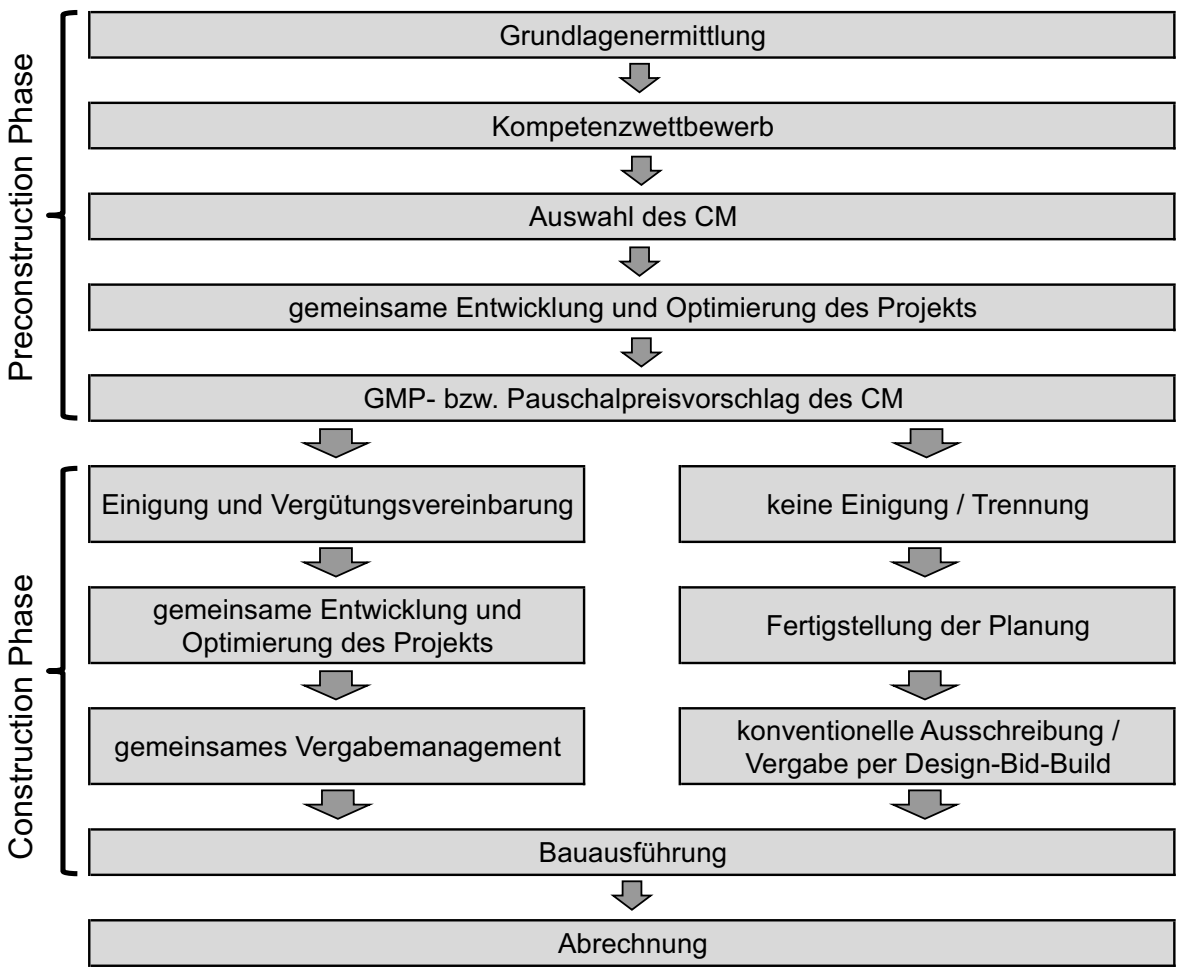

Abb. 1 Verfahrensablauf CM at risk

ersten Phase umfangreiche Projektmanagement- und Beratungsleistungen auf Honorarbasis. Hierbei berät er den AG bezüglich der Architektenplanung, überprüft deren technische Realisierbarkeit und bringt sein Know-How in Bezug auf Kosten und Termine ein.

Im Anschluss an die gemeinsame Optimierung der Planung wird der Abschluss eines Vertrages über die Bauleistungen angestrebt. In den meisten Fällen behält sich der AG das Recht vor, auf Basis der gemeinsam entwickelten Planung eine konventionelle Ausschreibung vorzunehmen, für den Fall, dass der Angebotspreis des CM nicht marktkonform erscheint (Gransberg und Shane 2010, S. 8).

Für die zweite Projektphase wird üblicherweise ein Bauvertrag mit Garantiertem Maximalpreis (GMP) abgeschlossen. Dieser sichert dem AG bei einem unveränderten Leistungssoll einen Maximalpreis zu und wird bei CM at risk als die zweckmäßigste Vertrags- und Vergütungsform angesehen (Gransberg und Shane 2010, S. $65 \mathrm{f}$.). Das Projektmanagement erfolgt hierbei gemeinschaftlich und transparent im open-books-Verfahren. Häufig werden im Rahmen der GMP-Vereinbarung Anreize vereinbart, die den CM bei einer Unterschreitung des GMP an den erzielten Einsparungen partizipieren lassen. Der idealtypische Ablauf einer Projektrealisierung mit $\mathrm{CM}$ at risk lässt sich dem Schema in Abb. 1 entnehmen.

An Stelle des klassischen Generalunternehmers kommt somit beim CM at risk ein hybrider Construction Manager/Generalunternehmer bzw. -übernehmer zum Einsatz 


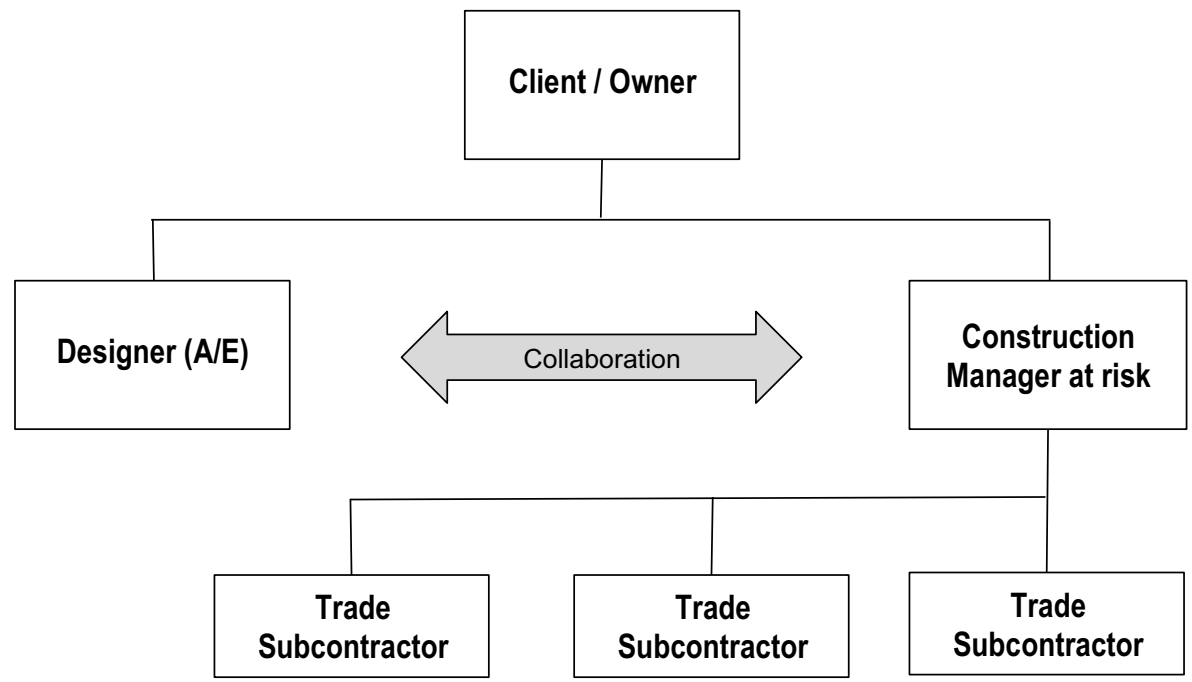

Abb. 2 Organisationsstruktur CM at risk (Quelle: Cunningham 2005)

(vgl. Cunningham 2005, S. 6). Dies begründet sich in der besonderen Konstellation, dass der CM während der Preconstruction Phase als Berater des AG und während der Construction Phase als Äquivalent eines Generalunternehmers agiert (Haltenhoff 1999, S. 70). Die Projektorganisationsstruktur gestaltet sich im Grundmodell des CM at risk entsprechend Abb. 2.

\section{Probleme in der Praxis}

Trotz umfangreicher Bemühungen zur Implementierung von CM at risk in der deutschen Bau- und Immobilienwirtschaft etwa seit der Jahrtausendwende kommt es bei der konkreten Umsetzung in der Praxis nach wie vor zu umfassenden Problemen (vgl. Heidemann 2010, S. 161; Eschenbruch und Racky 2008, S. $110 \mathrm{ff}$; Roland Berger Strategy Consultants 2008, S. 8.). Nicht zuletzt deswegen ist die Akzeptanz bei den verantwortlichen Entscheidungsträgern der Immobilienbeschaffung nach wie vor gering. Folgerichtig hat bisher auch noch keine signifikante Marktdurchdringung stattgefunden.

In der Literatur finden sich verschiedene Erklärungsansätze, warum bei der Immobilienbeschaffung trotz der erwiesenen Vorteile kooperativer Projektabwicklung immer noch stark auf konventionelle Projektorganisationsformen zurückgegriffen wird. Seitens der Auftraggeber herrscht häufig immer noch eine grundlegende Skepsis mangels Erfahrungen im Umgang mit alternativen Projektorganisationsformen. Zudem wird auf bestehende Probleme bzw. Unwissenheit bei der Ausgestaltung der Projektorganisationsformen sowohl in organisatorischer (ex ante) als auch vertraglicher (ex post) Hinsicht hingewiesen (Eschenbruch und Racky 2008, S. 110; Loskant 2010, S. 97; Acker 2006, S. 140). Häufig werden beispielsweise nur einzelne Bestandteile eines umfassenden Partnering-Konzeptes angewendet, deren partieller 
Einsatz jedoch nicht funktioniert (Schmolke 2008, S. 5; vgl. Ohler et al. 2012, Rdn 282). Mathoi weist darauf hin, dass Auftraggeber oft Verträge mit Maximalpreisvereinbarungen schließen, jedoch in praxi an Stelle der konsequenten Umsetzung des partnerschaftlich orientierten Charakters weiterhin die konfrontative Kultur der Bauprojektabwicklung praktizieren (Mathoi 2006, S. 80; ähnlich Ohler et al. 2012, Rdn 255).

Eine weitere Problematik wird darin gesehen, dass das Vergütungssystem häufig dem konventioneller Organisationsmodelle ähnelt. Es können somit trotz Partnerschaftsvereinbarungen win/lose-Situationen auftreten, die einen Partner dazu veranlassen, seine eigenen Ziele zu verfolgen (Schlabach 2013, S. 2). Bücker 2005 konstatiert, dass die Ausgestaltung der Vergabe und der Vertragsbedingungen eine herausragende Rolle spielen, um die Chancen des CM at risk zu nutzen und die Risiken zu verringern.

Die bisher in Deutschland praktizierten Gestaltungsformen von CM at risk bzw. daran angelehnter Wettbewerbsformen unterscheiden sich zum Teil ganz erheblich hinsichtlich ihres Organisationsaufbaus, des Verfahrensablaufs sowie der eingesetzten Instrumente zur Gestaltung und Steuerung der Wertschöpfungspartnerschaft. Diese Inhomogenität ist mutmaßlich eine wesentliche Ursache für den sehr unterschiedlichen Erfolg in der Praxis. So zeigte die Untersuchung zweier Pilotprojekte deutliche Unterschiede hinsichtlich der Effizienzsteigerungswirkungen immobilienwirtschaftlicher Wertschöpfungspartnerschaften in Abhängigkeit von den jeweiligen Strukturen im Projekt (Meyer und Pfnür 2015, S. 61). Der Schlüssel für eine erfolgreiche Implementierung liegt damit in der Optimierung und Standardisierung der organisatorischen und vertraglichen Ausgestaltung.

Dazu bedarf es der Offenlegung der organisationsimmanenten Wirkungszusammenhänge und der Identifikation der kritischen Erfolgsfaktoren dieser Organisationsform. Unabdingbare Voraussetzung hierzu ist zunächst eine umfassende Bestandsaufnahme der institutionellen Ausgestaltung von CM at risk in der Praxis.

\section{Empirische Untersuchung}

\subsection{Methodik der Datenerhebung}

Zur Feststellung des Status Quo von CM at risk in der deutschen Bau- und Immobilienwirtschaft wurde eine standardisierte Befragung von ausgewählten Experten durchgeführt.

Das entscheidende Kriterium bei der Auswahl der Experten war hierbei deren konkrete Praxiserfahrung bei der Projektabwicklung mit CM at risk; vorzugsweise bereits bei mehreren Projekten. Die Generierung geeigneter Interviewpartner erfolgte in mehreren Schritten und war aufgrund der nach wie vor verhältnismäßig geringen Verbreitung von $\mathrm{CM}$ at risk in der immobilienwirtschaftlichen Beschaffung relativ aufwändig. Um den potenziellen Interviewpartnern den Untersuchungsgegenstand näher zu bringen und den Ablauf der Interviews genauer zu erläutern, wurde eine umfassende Vorabinformation durchgeführt. Um einen möglichst reibungslosen Ablauf der Interviews und eine hohe Qualität und Vollständigkeit der Antworten zu 
gewährleisten, wurde der Fragebogen inkl. eines erläuternden Anschreibens vorab übermittelt.

Aus den etwa 150 getätigten Anfragen ergaben sich insgesamt 74 Experteninterviews. Diese hohe Quote ist wahrscheinlich auf die sehr aufwändige Recherche potentieller Interviewpartner im Vorfeld zurückzuführen. Gleichzeitig ist dies ein Indiz für die nach wie vor hohe Relevanz dieses Themas bei den befragten Institutionen, was u.a. auch durch ein entsprechendes Feedback während der Interviews wiederholt bestätigt wurde.

Sämtliche Gesprächsinhalte wurden während der Befragung handschriftlich protokolliert. Die Aufzeichnungen konnten im Anschluss an das Interview von den Experten zur Gegenprüfung eingesehen werden.

\subsection{Methodik der Datenauswertung}

Die Auswertung der im Rahmen der Interviews gewonnenen Daten erfolgte mit Hilfe der qualitativen Inhaltsanalyse. Sowohl die qualitative als auch die quantitative Sozialforschung haben gemeinsam, dass sie „,soziales Handeln deutend verstehen“ wollen, „um es in seinem Ablauf und in seinen Wirkungen ursächlich zu erklären“. Dabei wird sowohl untersucht, welche Faktoren ein bestimmtes Handeln mit einer gewissen Wahrscheinlichkeit hervorbringen als auch wie die gefundenen Faktoren das Resultat erzeugen (Gläser und Laudel 2010, S. 25).

Bezogen auf den hier zugrunde liegenden Untersuchungsgegenstand, die Projektorganisation beim CM at risk, bedeutet das, dass die kausalen Zusammenhänge zwischen der institutionellen Ausgestaltung der Projektorganisation (Ursache) und dem Handeln der Akteure (Wirkung) systematisch erschlossen und offengelegt werden sollen. Gleichzeitig soll der Kausalmechanismus identifiziert werden, also warum ein bestimmter Faktor zu einer gewissen Wahrscheinlichkeit eine bestimmte Reaktion hervorruft.

Die gegenständliche Forschungsstrategie ist als überwiegend theoriegenerierender, qualitativer Ansatz zu klassifizieren (Bogner et al. 2014, S. 22 ff.). Hierbei wird anhand einer detaillierten Analyse eines oder weniger Fälle nach den Kausalmechanismen gesucht, die unter bestimmten Bedingungen bestimmte Effekte hervorbringen. Eine Schwäche dieser mechanismenorientierten Strategie liegt darin, dass die Verbreitung dieser Bedingungen nicht ermittelt werden kann (Gläser und Laudel 2010, S. 26). Diese lässt sich wiederum mit dem Ansatz der relationsorientierten, quantitativen Strategie bestimmen.

Diese Methode lässt sich aufgrund einer nicht ausreichenden statistischen Signifikanz auf den untersuchungsgegenständlichen Sachverhalt nur bedingt anwenden, kommt jedoch komplementär zur Anwendung. In der Forschungspraxis erfolgt in den meisten Fällen eine Kombination beider Ansätze, da es gar keine rein quantitativen (nicht mit Interpretationen verbundenen) Methoden gibt und auch qualitative Studien häufig mit Zahlen oder Mengenangaben arbeiten (Gläser und Laudel 2010, S. 25).

Mit Hilfe der qualitativen Inhaltsanalyse wurde das aus den Interviews gewonnene Wissen der Experten konzeptualisiert (Bogner et al. 2014, S. 72). Im Rahmen eines vierstufigen Auswertungskonzeptes der renommierten Sozialforscher Gläser 
und Laudel wurden die gesammelten Informationen systematisch analysiert und verglichen, um die inhärenten Kausalzusammenhänge aufzudecken.

Entsprechend des v. g. Konzeptes wurden vom Verfasser die nachfolgenden Bearbeitungsschritte vollzogen:

1. Vorbereitung der Extraktion,

2. Extraktion,

3. Aufbereitung,

4. Auswertung.

Die wesentlichen, mit Hilfe der qualitativen Inhaltsanalyse aus den Experteninterviews gewonnenen Erkenntnisse werden nachfolgend im Detail zusammenfassend dargestellt.

\section{Ergebnisse}

\subsection{Zusammensetzung der Befragten}

Der erste Teil des Interviewfragebogens widmete sich der Erfassung allgemeiner Angaben zur Person und Institution des Interviewpartners sowie den Grunddaten zum untersuchungsgegenständlichen Projekt.

Ein Ziel der Befragung war es, eine möglichst breit angelegte Erhebung durchzuführen, welche alle wesentlichen Beteiligten innerhalb der Projektorganisation in einem möglichst ausgewogenen Verhältnis zwischen Vertretern der Auftraggeberund Auftragnehmerseite umfasst. Hierdurch sollte eine so objektiv wie mögliche Darstellung von CM at risk in der Praxis gewährleistet werden, welche die verschiedenen Befindlichkeiten aller Projektbeteiligten erfasst. Gleichzeitig sollten durch diese Vorgehensweise etwaige Idealisierungen durch einzelne Akteurs- und Interessensgruppen aufgedeckt und somit eine Verfälschung der Ergebnisse vermieden werden.

Abb. 3 zeigt die Gliederung der Befragten nach der Art ihrer Institution. Die Hauptakteursgruppen der Auftraggeber und Bauunternehmen nehmen gemeinsam einen Anteil von $80 \%$ ein. Die verbleibenden Anteile verteilen sich auf Projektmanager/Consultants mit $12 \%$ und Architekten mit $8 \%$, d.h. Erfüllungsgehilfen des AG. Die Zusammensetzung der befragten Experten zeigt, dass die Bauunternehmen mit 51\% und Auftraggeber mit 29\% deutlich überrepräsentiert sind. Dies liegt im Wesentlichen daran, dass diese die Hauptakteure innerhalb der Projektorganisation sind und dadurch ein erhöhtes Interesse am Untersuchungsgegenstand haben dürften. Gleichzeitig verfügen diese beiden Akteursgruppen, speziell die Bauunternehmen, in der Breite über die umfangreichste Expertise bei der Umsetzung von CM at risk bzw. daran angelehnter Wettbewerbsmodelle in der Praxis.

Unter der Akteursgruppe der Auftraggeber befanden sich zehn Projektentwickler, drei institutionelle sowie acht sonstige Auftraggeber, worunter u. a. die öffentliche Hand und Industrieunternehmen aggregiert wurden. Bei der überwiegenden Mehrheit handelte es sich somit um professionelle Akteure, nicht um Einmalbauherrn. 
Abb. 3 Art der Institution

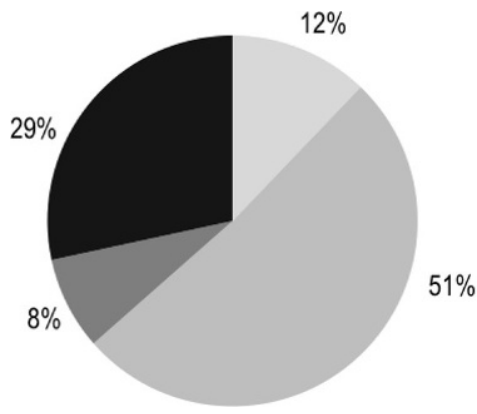

Projektmanager/ Consultants

Bauunternehmen

- Architekten

Auftraggeber

Bauleiter/

Oberbauleiter

Abb. 4 Position der Interviewpartner im Unternehmen

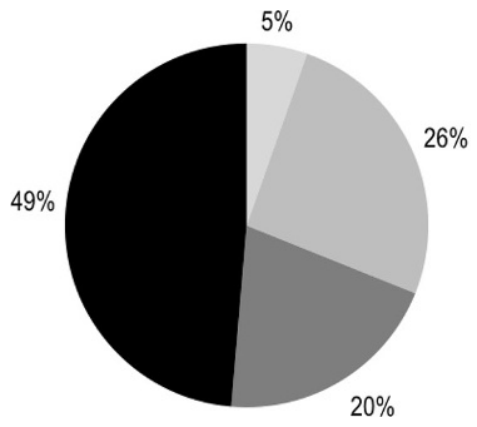

Projektleiter

- Stabsfunktion

- Geschäftsführerl Prokurist

Unter den Auftraggebern befanden sich lediglich zwei öffentliche bzw. halböffentliche Institutionen. Dies ist ein Indiz dafür, dass alternative Projektorganisationsformen im öffentlichen Sektor nach wie vor stark unterrepräsentiert sind, was im Wesentlichen den Einschränkungen durch das deutsche Vergaberecht geschuldet sein dürfte. Durch die Vergaberechtsreform 2016 und die Einführung der neuen Vergabeart der „Innovationspartnerschaft“" wurde die Realisierung von Projekten mit Hilfe alternativer Projektorganisationsformen für öffentliche Auftraggeber etwas erleichtert.

Unter den Befragten der Bauunternehmen waren 11 Akteure für Baukonzerne sowie 27 für mittelständische Unternehmen tätig. Dies verdeutlicht, dass CM at risk nicht ausschließlich den Baukonzernen vorbehalten ist, sondern auch von vielen Mittelständlern als alternatives Projektorganisationsmodell genutzt wird, um dem Kunden einen erkennbaren Mehrwert anbieten zu können und sich dadurch von den Mitbewerbern abzugrenzen.

Abb. 4 zeigt die Position der Interviewpartner innerhalb ihrer Unternehmen. Unter den Befragten befanden sich demnach überwiegend Vertreter der oberen Managementebene, also mit einer strategischen, nicht-operativen Funktion. Hierzu zählten Geschäftsführer, Prokuristen, Bereichs- und Niederlassungsleiter sowie Personen in verschiedenen Stabsfunktionen. Daneben wurden auch Experten in operativer Funktion befragt, wie Projektleiter, Oberbauleiter und Bauleiter.

Auch in dieser Hinsicht deckt die Erhebung das mögliche Befragungsspektrum weitgehend $a b$. Als möglicher Grund für das Überwiegen von Befragten mit strategischer, nicht-operativer Funktion im Unternehmen kann die tendenziell strategische 
Dimension alternativer Projektabwicklungsmodelle angesehen werden. Darüber hinaus sollten häufig keine operativen Kräfte interviewt werden, um das laufende Projektgeschäft nach Möglichkeit nicht zu stören.

\subsection{Art und Umfang der Projekte}

Die 74 Experten wurden zu insgesamt 51 verschiedenen Projekten befragt. Zu einigen Projekten wurden demnach unterschiedliche Projektbeteiligte interviewt, so dass es zu einzelnen Dopplungen kam. Darüber hinaus waren einige Interviewpartner aus Gründen der Vertraulichkeit oder mangels Detailwissen nicht bereit bzw. in der Lage, über ein konkretes Projekt Auskunft zu erteilen. Häufig traf dies auf die Befragten aus dem Topmanagement der Konzerne zu. In diesem Fall wurde die Befragung ohne konkreten Projektbezug durchgeführt. Die aus diesen Interviews gewonnenen Erkenntnisse waren auch projektunabhängig in vollem Umfang zu verwerten, da gerade auch Querschnittswissen aus mehreren Projekten wertvolle Erkenntnisse über den Untersuchungsgegenstand liefern kann (vgl. Gläser und Laudel 2010, S. 271). Abb. 5 gibt einen Überblick über das Projektvolumen der untersuchten Projekte.

$\mathrm{CM}$ at risk wird demzufolge bei Projekten nahezu jeglicher Größenordnung praktiziert, wobei die Untergrenze bei den untersuchten Projekten bei einer Investitionssumme von 2,5 Mio. $€$ und die Obergrenze bei ca. 300 Mio. $€$ lag. Die v. g. Untergrenze i.H. v. 2,5 Mio. $€$ kann gleichzeitig auch in etwa als kritische Mindestprojektgröße in Bezug auf die Effizienz von CM at risk angesehen werden.

Die Einsatzhäufigkeit von $\mathrm{CM}$ at risk nimmt mit steigendem Projektvolumen zu. Dieser Zusammenhang begründet sich darin, dass mit steigender Investitionssumme üblicherweise auch eine steigende Projektkomplexität einhergeht. Dies wiederum lässt die Anwendung von Wertschöpfungspartnerschaften als alternatives Organisationsmodell bei der Immobilienbeschaffung effizient werden. Dieses Bewusstsein ist offenbar bei den ökonomischen Akteuren präsent und zeigt sich in den Ergebnissen der Befragung.

Die Art der untersuchten Projekte ist relativ vielschichtig. Insgesamt die Hälfte aller Projekte entfällt mit $27 \%$ bzw. 23\% auf die Projektarten Büro und Wohnen. Die restlichen Projekte verteilen sich verhältnismäßig gleichmäßig zu 16\% auf Retail, $18 \%$ auf Industrieprojekte sowie $16 \%$ auf sonstige Projekte, wie z. B. Sonderimmobilien, Infrastrukturprojekte oder Gewerbeimmobilien.

Die Entscheidungsträger auf Auftraggeberseite erachten demzufolge den Einsatz von CM at risk als vorteilhaft und anwendbar für ein breites Spektrum unterschiedlicher Projektarten. Abb. 6 zeigt die Untergliederung der untersuchten Projekte nach der Projektart.

\subsection{Die organisatorische und vertragliche Ausgestaltung}

Der zweite Teil des Interviewfragebogens hatte zum Ziel, die organisatorische und vertragliche Ausgestaltung von CM at risk bzw. daran angelehnter Projektorganisationsformen in der deutschen Bau- und Immobilienwirtschaft zu untersuchen. Darauf aufbauend lässt sich eine Gegenüberstellung der wesentlichen Strukturen und Gestaltungsparameter mit dem in Nordamerika praktizierten Grundmodell des CM at risk 
Abb. 5 Projektvolumen der untersuchten Projekte

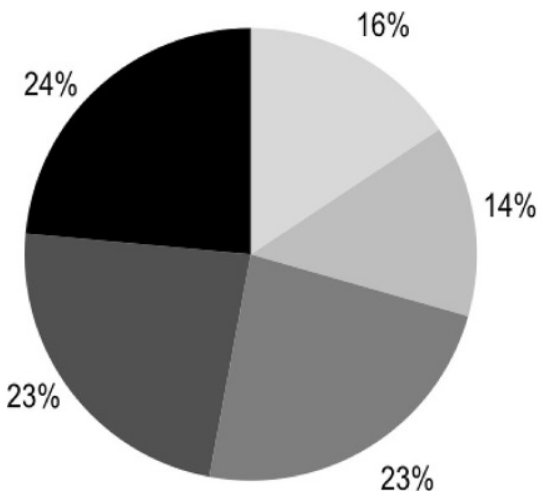

$0-5$ Mio $€$

플

= 10-20 Mio€

20-50 Mio€

$>50$ Mio $€$

- Büro

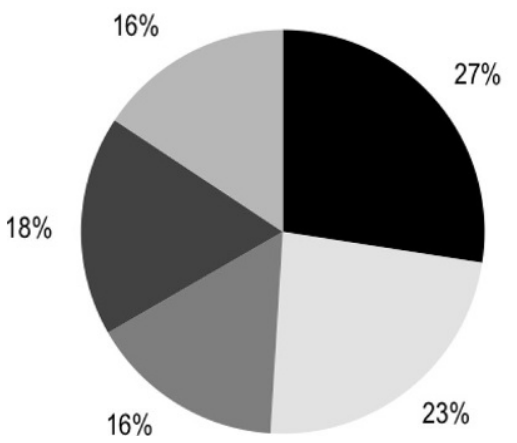

Wohnen

- Retail

- Industrie

Sonstige

vornehmen. Dieser Abgleich ermöglicht grundlegende Rückschlüsse auf modellimmanente Ineffizienzen sowie potenzielle Konfliktpotenziale und Problemfelder. $\mathrm{Ob}$ und inwieweit sich die konkrete Ausgestaltung der organisatorischen und vertraglichen Gestaltungsparameter auf das Verhältnis der Projektbeteiligten während der Projektabwicklung auswirkt, wird anschließend im dritten Teil des Fragebogens untersucht.

Die strukturierte Auswertung der Interviewdaten ergab, dass die in Deutschland praktizierten Abwicklungsmodelle in wesentlichen organisatorischen und vertraglichen Handlungsfeldern fast durchgängig signifikante Abweichungen gegenüber dem in den USA praktizierten CM at risk aufweisen.

So ist tendenziell festzustellen, dass beim CM at risk in der deutschen Bau- und Immobilienwirtschaft sehr häufig eine mehr oder weniger ausgeprägte Parallelorganisation praktiziert wird, indem neben dem CM eine zusätzliche Projektsteuerung auf AG-Seite ins Projekt eingebunden wird. Bei insgesamt 23 von 51 untersuchten Projekten wurde vom AG neben dem CM ein separater Projektsteuerer bzw. Controller beauftragt. Durch die häufige Parallelorganisation unterscheiden sich die in Deutschland praktizierten Abwicklungsformen somit vielfach bereits hinsichtlich ihrer Projektstruktur vom amerikanischen Grundmodell des CM at risk. Die Einbindung eines zusätzlichen Interessenvertreters auf Auftraggeberseite hat abhängig von dessen Kompetenzen sowie der Integrationstiefe mutmaßlich einen signifikanten Einfluss auf das Vertrauensverhältnis zwischen den Projektbeteiligten. Das parallele 
Abb. 7 Vergabekriterien bei den untersuchten Projekten

Abb. 8 Zeitpunkt des Projekteintritts des CM
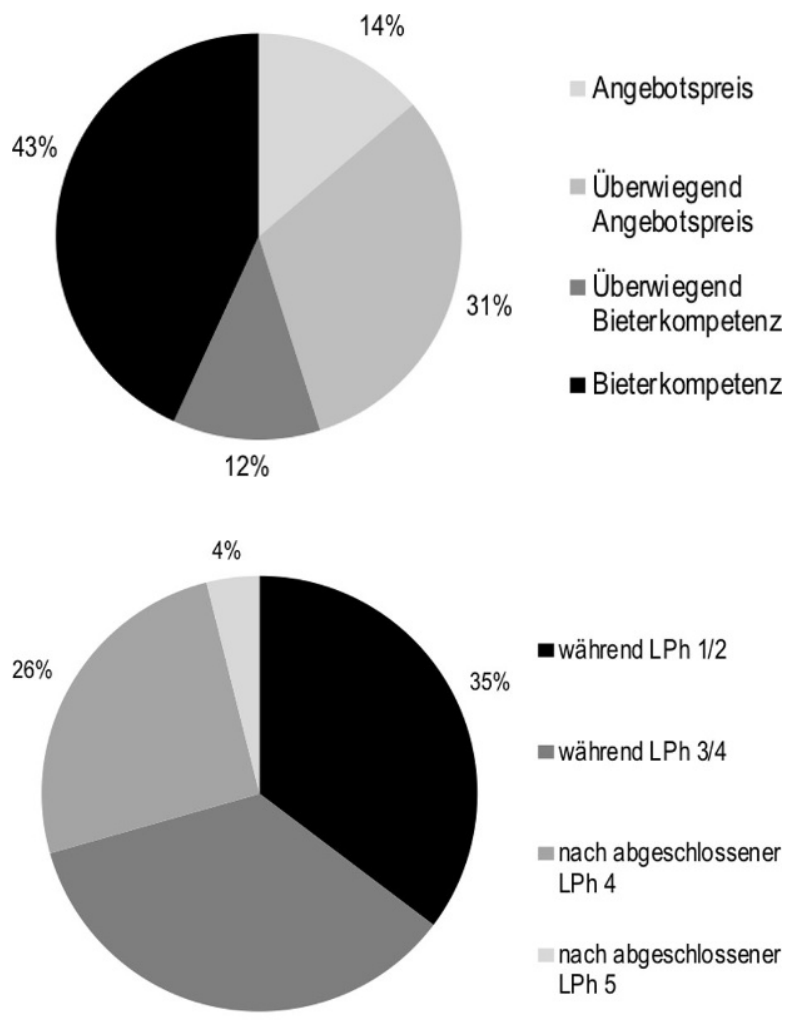

- während LPh $1 / 2$

während LPh 3/4

nach abgeschlossener LPh 4

nach abgeschlossener LPh 5

$35 \%$

Management von Prozessen wirkt sich tendenziell negativ auf den Value for Money und damit die Effizienz der Wertschöpfungspartnerschaft aus.

Darüber hinaus hat die Untersuchung gezeigt, dass die Vergabe im Gegensatz zum Grundmodell des amerikanischen CM at risk häufig ausschließlich bzw. überwiegend auf Basis des Angebotspreises vorgenommen wird und somit preisliche Kriterien gegenüber der Bieterkompetenz dominieren (vgl. Abb. 7). Bei 23 Projekten, mit einem Anteil von 45\% also nahezu der Hälfte aller untersuchten Projekte, war demzufolge nicht die Bieterkompetenz, sondern der Angebotspreis das entscheidende bzw. überwiegende Kriterium bei der Vergabe.

Die Ausgestaltung des Vergabewettbewerbs widerspricht damit in einer Vielzahl der Fälle dem Grundkonzept von CM at risk, welches grundsätzlich einen Kompetenzwettbewerb zur Auswahl des Projektpartners vorsieht. Die Vergabe nach preislichen Kriterien analog der Vergabe bei konventionellen Projektorganisationsformen hat mutmaßlich einen signifikanten Einfluss auf das Verhältnis zwischen den Projektbeteiligten. Auch an dieser Stelle wird somit deutlich, dass in der Praxis der deutschen Bau- und Immobilienwirtschaft vielfach eine für $\mathrm{CM}$ at risk ungeeignete institutionelle Ausgestaltung von Projektorganisation und Projektablauf vorgenommen wird. 
Die häufig praktizierte Vergabe mit einer Übergewichtung von preislichen Kriterien korrespondiert auch mit der Tatsache, dass der Projekteintritt des CM in 26\% der untersuchten Projekte erst nach bereits abgeschlossener Leistungsphase (LPh) 4 der Honorarordnung für Architekten und Ingenieure (HOAI) erfolgte (vgl. Abb. 8). Bei zwei Projekten wurde der CM sogar erst nach Abschluss der LPh 5 der HOAI beauftragt.

Die für $\mathrm{CM}$ at risk charakteristische Preconstruction Phase wird bei einem derart späten Projekteintritt des AN zwangsläufig auf einen verhältnismäßig kurzen Zeitraum während der Ausführungsplanung reduziert bzw. macht sogar einen Rückgriff auf die bereits abgeschlossene Ausführungsplanung notwendig. Die dadurch bereits stark eingeschränkte Möglichkeit zur Planungsoptimierung bzw. der resultierende Mehraufwand durch den notwendigen Rückgriff auf bereits abgeschlossene Planungsphasen beeinträchtigt die Effizienz der Wertschöpfungspartnerschaft und wirkt sich damit negativ auf den Value for Money aus. Diese Form der institutionellen Ausgestaltung von CM at risk in der Praxis lässt sich somit ebenfalls als ungeeignet und ineffizient bezeichnen.

Durch die häufig späte Einbindung ins Projekt erhält der CM in vielen Fällen keine Vergütung für seine Leistungen in der Preconstruction Phase, da die AGSeite keinen oder nur einen geringen vergütungswürdigen Input erwartet und der dem CM entstehende Aufwand daher üblicherweise als herkömmlicher Aufwand der Projektakquise angesehen wird.

Bei insgesamt 19 Projekten (37\%) erhielt der CM keinerlei Vergütung für seine Leistungen in der Preconstruction Phase. Bei den verbleibenden 32 Projekten (63\%) erhielt der CM zwar eine Vergütung, die jedoch überwiegend nur für den Fall der Nichtbeauftragung mit der Construction Phase gewährt wurde, d.h. im Auftragsfall in der Auftragssumme mit aufging. Eine von der Beauftragung der Bauleistung unabhängige Vergütung für die Preconstruction Services wurde somit nur in wenigen Fällen vorgenommen.

Sofern überhaupt eine Vergütung für die Preconstruction Phase gewährt wurde, wurde diese lediglich in 19\% von den Interviewpartnern als kostendeckend bezeichnet. Das bedeutet, dass in $81 \%$ der Projekte lediglich eine symbolische Entschädigung gezahlt wurde.

Die vorstehend erläuterten Erkenntnisse aus den Experteninterviews in Hinblick auf die Vergütung des $\mathrm{CM}$ in der Preconstruction Phase sind in der nachfolgenden Abb. 9 dargestellt.

Die oft späte Einbindung des CM ins Projekt und die mangelnde Bereitschaft zur Vergütung der Preconstruction Services korrespondiert auch mit einem oft stark reduzierten Leistungsumfang verglichen mit den Leistungen des CM im angloamerikanischen Raum.

In etwa einem Drittel der untersuchten Projekte wurden für CM at risk charakteristische Leistungen der Preconstruction Phase wie z.B. das Aufstellen der Kostenschätzung und des Projektterminplans nicht vom CM, sondern federführend durch den AG bzw. dessen Erfüllungsgehilfen erbracht. In diesen Fällen war häufig eine ausgeprägte Parallelorganisation zu beobachten, indem der CM nach Projekteintritt nochmals eigene Ermittlungen vornahm, die der nachträglichen Überprüfung und Anpassung der bereits AG-seitig erstellten Unterlagen diente. 
Abb. 9 Vergütung für Preconstruction Services

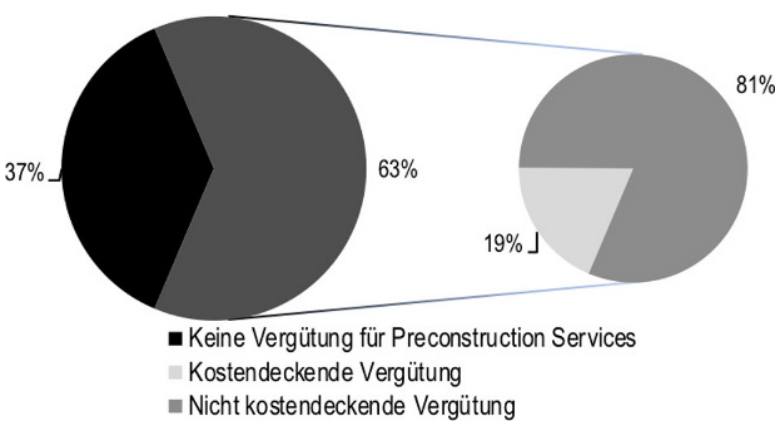

Abb. 10 Klassifizierung nach Projektorganisationsformen

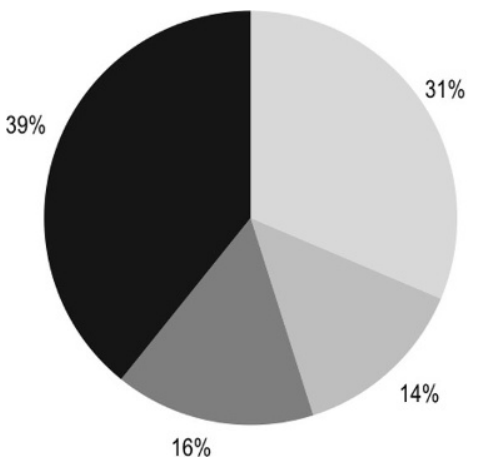

Design-Bid-Build (DBB)

Design-Build (DB)

- CM at risk (per

Definition)

- $\mathrm{CM}$ at risk (LPh 5)

Die für $\mathrm{CM}$ at risk charakteristische, kontinuierliche Bewertung der Planung in Bezug auf Kosten und Termine sowie die Überprüfung der Realisierbarkeit der Planung und Terminvorgaben wurde zum Teil gar nicht bzw. aufgrund der späten Einbindung ins Projekt nur in stark reduziertem Umfang vom CM vorgenommen. Diese Form der institutionellen Ausgestaltung von CM at risk in der Praxis lässt erhebliche Wertschöpfungspotenziale ungenutzt, die sich durch die frühzeitige Integration des $\mathrm{CM}$ bieten und ist daher als ungeeignet und ineffizient zu betrachten.

Die vorstehend erläuterten Projektcharakteristika zeigen, dass die institutionelle Ausgestaltung von $\mathrm{CM}$ at risk in der Praxis der deutschen Bau- und Immobilienwirtschaft mitunter erheblich vom US-amerikanischen Modell abweicht. Nach Kenig (2011) ist CM at risk anhand von zwei Kriterien eindeutig zu identifizieren und von den übrigen Projektorganisationsformen abzugrenzen. Kennzeichnend für CM at risk ist demnach die getrennte Vergabe von Planung und Ausführung sowie die Vergabe nach Kompetenzkriterien, d.h. ohne die Berücksichtigung der Baukosten als Wertungskriterium (Kenig 2011, S. 6). Die frühzeitige Einbindung des CM in der frühen Planungsphase wird hierbei als systembedingte Notwendigkeit vorausgesetzt.

Bei konsequenter Anwendung dieser Abgrenzungssystematik lassen sich nur 16\% aller untersuchten Projekte per Definition als ,echtes“ CM at risk klassifizieren. Dies liegt daran, dass häufig die für $\mathrm{CM}$ at risk charakteristische Trennung zwischen Planung und Ausführung nicht konsequent verfolgt wurde, da die Erstellung der Ausführungsplanung in der Mehrzahl der Fälle an den CM übertragen wurde. 
Bei einer etwas erweiterten definitorischen Auslegung können 39\% aller Projekte als „CM at risk mit LPh 5“ eingeordnet werden. Das bedeutet, dass der CM im Rahmen eines Kompetenzwettbewerbs ausgewählt und beauftragt wurde und die Planungsleistungen bis einschließlich HOAI LPh 4 durch einen AG-seitigen Architekten erbracht wurden. Die Ausführungsplanung gemäß HOAI LPh 5 wurde anschließend im Rahmen des Bauvertrages an den CM übertragen. Diese Ausgestaltungsform hat ihren Ursprung in der in Deutschland häufig praktizierten Generalunternehmervergabe mit Übertragung der Ausführungsplanung an den Generalunternehmer.

Aufgrund einer ausschließlich bzw. überwiegend preisbasierten Vergabe waren $31 \%$ der untersuchten Projekte definitionsgemäß als konventionelle Projektabwicklung mit Generalunternehmer einzuordnen (Design-Bid-Build). In diesen Fällen erfolgte die Projektabwicklung zumindest partiell mit einem partnerschaftlichen Ansatz. Diese Projekte sind gegenüber der klassischen Projektabwicklung überwiegend durch eine etwas frühere Einbindung des CM in den späten Planungsphasen gekennzeichnet, so dass zumindest in Teilen Ausführungs-Know-How zur Optimierung der Planung genutzt werden kann.

Bei weiteren $14 \%$ der untersuchten Projekte handelte es sich aufgrund der gemeinsamen Vergabe von Planungs- und Bauleistungen innerhalb eines Vertrages per Definition um eine Projektabwicklungsform mit einem Totalunternehmer (DesignBuild). Auch die Realisierung dieser Projekte erfolgte mit einem partnerschaftlichen Grundansatz.

Die vorstehend erläuterte Klassifizierung der untersuchten Projekte nach Projektorganisationsformen entsprechend der Abgrenzungssystematik nach Kenig (2011) ist in Abb. 10 grafisch dargestellt. Das Ergebnis zeigt, dass es sich bei den als CM at risk konzipierten Projektorganisationsformen in der deutschen Bau- und Immobilienwirtschaft aufgrund ihrer institutionellen Ausgestaltung überwiegend nicht um $\mathrm{CM}$ at risk im definitorischen Sinn handelt.

\subsection{Problemfelder und Erfolgsfaktoren}

Teil 3 des Interviewfragebogens hatte zum Ziel, Problemfelder und Erfolgsfaktoren von $\mathrm{CM}$ at risk zu identifizieren. Dabei sollten die komplexen Kausalzusammenhänge zwischen der organisatorischen und vertraglichen Ausgestaltung von CM at risk und dem Verhalten der ökonomischen Akteure während der Projektabwicklung offengelegt werden.

\subsubsection{Organisation des Ausschreibungs- und Vergabeprozesses}

Als einer der kritischen Erfolgsfaktoren beim CM at risk wurde die Gestaltung des Ausschreibungs- und Vergabeprozesses identifiziert. Mit ca. 89\% war eine deutliche Mehrheit der befragten Experten der Auffassung, dass die Einbindung des CM möglichst frühzeitig erfolgen sollte, um einen maximalen Einfluss auf die Planung zu gewährleisten, Optimierungen gleich im Zuge des regulären Planungsprozesses integrieren zu können und dadurch keine unnötigen Kosten für nachträgliche Umplanungen zu erzeugen. Tendenziell war festzustellen, dass die Bauunternehmen eine frühzeitigere Einbindung als erforderlich erachteten als die übrigen Befrag- 
ten. Nach Auffassung einiger Experten bietet die frühzeitige Integration des CM in den Planungsprozess den Vorteil, dass eine Vielzahl von Projektrisiken gemeinsam eliminiert werden kann.

Die häufig späte Einbindung des CM führt in der Praxis zu einer Vielzahl von Problemen. In mehreren Projekten war die Planung bereits so stark fixiert, so dass kaum noch Optimierungen vorgenommen werden konnten, ohne erhebliche nachträgliche Planungsänderungen zu verursachen. Vergütungsstreitigkeiten aus einer nicht einheitlichen Bau-Soll-Definition wurden ebenfalls auf den späten Projekteintritt des CM zurückgeführt.

Die starke Korrelation zwischen dem Zeitpunkt der Integration des CM in das Projekt und dem Verhältnis zwischen den Projektbeteiligten wurde mehrfach auch dadurch bestätigt, dass die Befragten von einem positiven und weitestgehend konfliktfreien Projektverlauf berichteten, den sie gerade auf die frühzeitige Einbindung des CM zurückführten.

Neben der möglichst frühen Integration des CM war mit $91 \%$ ebenfalls eine deutliche Mehrheit der Befragten der Auffassung, dass die Vergabe überwiegend nach Kompetenzkriterien erfolgen sollte. Dies wurde größtenteils damit begründet, dass der Kompetenzwettbewerb eine gemeinsame Vertrauensbasis für die Projektpartnerschaft bildet und Kostenparameter in den frühen Planungsphasen ohnehin wenig aussagekräftig sind. Die Befragungsergebnisse zeigten eine positive Korrelation zwischen kompetenzbasierter Vergabe und einer weitestgehend konfliktfreien Projektabwicklung. Die Vergabe nach Kompetenzkriterien hat demzufolge einen erkennbar positiven Einfluss auf das Verhältnis zwischen den Projektbeteiligten.

\subsubsection{Kooperation zwischen Architekt und AN}

Mit 95\% sahen nahezu alle Experten die Kooperation zwischen dem Architekten und dem CM als einen kritischen Erfolgsfaktor beim CM at risk an. Die enge Kooperation zwischen diesen beiden Akteuren wird als Grundvoraussetzung für einen effizienten Planungsoptimierungsprozess angesehen, der ein charakteristisches Element des CM at risk ist. Ein effizientes Value Engineering ist wiederum die Voraussetzung, um die Effizienzsteigerungspotenziale der Wertschöpfungspartnerschaft bestmöglich zu nutzen.

In mehreren Projekten kam es zu erheblichen Problemen durch eine unzureichende Kooperation zwischen Architekt und CM. In ca. 85\% dieser Fälle war der CM erst sehr spät ins Projekt eingebunden worden, so dass die Planung bereits entsprechend weit fortgeschritten war. Auf die negative Korrelation zwischen einer späten Einbindung des CM und der Kooperationsbeziehung wurde von einer Reihe der Befragten explizit hingewiesen.

\subsubsection{Trennung von Planung und Ausführung}

Uneinig waren sich die Befragten bzgl. der konsequenten Trennung von Planung und Ausführung über alle Leistungsphasen der HOAI. Sowohl die durchgängige Trennung von Planung und Ausführung über alle Leistungsphasen der HOAI als auch die Übertragung der Planungsverantwortung auf den CM ab der LPh 5 der 
HOAI führten in der Praxis zu zufriedenstellenden Ergebnissen. Konkrete Probleme während der Projektabwicklung, die ursächlich auf diese Thematik zurückzuführen waren, wurden von den Experten nicht genannt. Aus der Gesamtheit der Interviewdaten ließ sich ebenfalls keine erhöhte Konfliktanfälligkeit einer bestimmten Ausgestaltungsvariante ableiten.

\subsubsection{Vergütung in der Preconstruction Phase}

Mit 82\% war die Mehrheit der Interviewpartner der Auffassung, dass der CM eine Vergütung für seine Leistungen in der Preconstruction Phase erhalten sollte. Nahezu alle Bauunternehmer waren der Ansicht, dass dem CM für die Leistungen in der Preconstruction Phase eine Vergütung gewährt werden sollte. Mit einem Anteil von $67 \%$ war jedoch auch ein relativ hoher Anteil der übrigen Befragten dieser Meinung.

Ein effizientes Value Engineering setzt nach Auffassung der befragten Experten ein umfassendes Engagement des CM voraus, welches jedoch nur bei einer entsprechenden Honorierung zu erwarten ist. Die Vergütung in der Preconstruction Phase schafft darüber hinaus „,eine Vertrauensbasis“ zwischen den beteiligten ökonomischen Akteuren und wirkt sich damit positiv auf die Effizienz der Wertschöpfungspartnerschaft aus.

\subsubsection{Erbringung von Eigenleistung durch den AN}

Nur eine Minderheit von 14\% der befragten Experten sahen die Erbringung von Eigenleistung durch den CM als problematisch an. Diese Problematik wurde nahezu ausschließlich seitens der Auftraggeber gesehen. Die deutliche Mehrheit war dagegen der Auffassung, dass die Marktkonformität des Eigenleistungsanteils über ein Baukosten-Benchmarking oder das Einstellen in den Wettbewerb überprüft werden kann. Mehrfach wurde ein hoher Eigenleistungsanteil auch als Indiz für eine hohe Ausführungskompetenz seitens des CM genannt.

Konkrete Probleme während der Projektabwicklung traten im Zusammenhang mit der Erbringung von Eigenleistung durch den CM lediglich in zwei Projekten auf. Dies wurde von den Experten in beiden Fällen darauf zurückgeführt, dass keine Pauschalierung des Eigenleistungsanteils vorgenommen worden war. Hier waren Vergütungsstreitigkeiten die Folge, die das Verhältnis zwischen den Vertragspartnern belasteten. Die Vereinbarung vertraglicher Regelungsmechanismen bzgl. der Vergabe von Leistungen an verbundene Unternehmen wird überwiegend als notwendig und sinnvoll erachtet und hat sich nach Auffassung der Befragten in der Praxis bewährt.

\subsubsection{Die Rolle des AG im Projekt}

Als ein weiterer kritischer Erfolgsfaktor beim CM at risk wurde die Rolle des AG im Projekt identifiziert. Mit 95\% waren sich nahezu alle befragten Experten einig, dass dieser eine ganz wesentliche Schlüsselposition innerhalb der Projektorganisation einnimmt. Der AG muss nach Auffassung der Experten seine Ziele definieren und mit seinen Entscheidungen sämtliche Prozesse zielgerichtet leiten. Um dieser 
Aufgabe gerecht werden zu können, muss er sich aktiv in das Projekt einbringen, um informierte Entscheidungen treffen zu können.

In den untersuchten Projekten war eine starke Korrelation zwischen der Integrationstiefe des AG und dem Verhältnis der Projektbeteiligten während der Projektrealisierung festzustellen. Je intensiver der AG in das Projekt eingebunden wurde, desto seltener kam es zu Problemen und Konfliktsituationen. Diese Abhängigkeit bestätigte sich auch dadurch, dass speziell bei solchen Projekten weitreichende Konflikte auftraten, bei denen der AG eine eher passive Rolle einnahm.

Die mangelnde Entscheidungsfähigkeit des AG war in mehreren Projekten die Ursache dafür, dass es zu wesentlichen Konflikten zwischen den Vertragspartnern während der Projektabwicklung kam. Als Ursache hierfür wurde mehrfach eine zu komplexe, hierarchische Struktur innerhalb der AG-Organisation sowie die Einbindung externer Consultants identifiziert. Komplexe AG-Strukturen waren häufig bei öffentlichen Auftraggebern sowie Großkonzernen anzutreffen und standen einer effizienten Projektabwicklung mit CM at risk tendenziell entgegen.

\subsubsection{Das gemeinsame Projektcontrolling}

Mit 97\% waren fast alle Experten der Auffassung, dass auch das gemeinsame Projektcontrolling ein wesentliches Element innerhalb der Projektorganisation beim CM at risk ist, so dass es sich dabei ebenfalls um einen kritischen Erfolgsfaktor handelt. Die transparente Projektabwicklung über open books schafft nach Meinung der Befragten Vertrauen, ermöglicht Kontrolle und senkt somit das Konfliktpotenzial. Das gemeinsame Projektcontrolling fördert damit die Kooperation zwischen den ökonomischen Akteuren und wirkt sich positiv auf die Effizienz der Wertschöpfungspartnerschaft aus.

Eine mangelnde Transparenz wurde mehrfach als Ursache für Probleme in der Projektabwicklung identifiziert. Ganz deutlich wurde dieser Zusammenhang in einem konkreten Projekt, als nach der erfolgreichen Preconstruction Phase mit einem gemeinsamen und damit transparenten Projektcontrolling für die Construction Phase ein Pauschalvertrag vereinbart wurde. Durch das Schließen der Bücher kam es zu einem Systembruch, der den Rückfall in die konventionelle, konfliktäre Projektabwicklung zur Folge hatte. Gleichzeitig führte die dann ausgeprägte Parallelorganisation zu gegenseitigem Misstrauen und damit zu erheblichen Problemen im Projekt.

\subsubsection{Die Vergabe der Nachunternehmerleistungen}

Die überwiegende Mehrheit von 93\% aller befragten Experten war der Auffassung, dass die Vergaben an die Nachunternehmer (NU) unter der Voraussetzung einer ausreichenden Transparenz weitestgehend reibungslos ablaufen. Die Ausdehnung der Wertschöpfungspartnerschaft auf die Nachunternehmerebene durch deren Einbindung in den planungsbegleitenden Optimierungsprozess steigert die Effizienz der Wertschöpfungspartnerschaft und ,hat sich in der Praxis bewährt“. Dies betrifft insbesondere die technisch anspruchsvollen Schlüsselgewerke.

Konkrete Probleme im Zusammenhang mit der Vergabe der NU-Leistungen konnten lediglich in wenigen Projekten identifiziert werden. Ursache hierfür waren unter- 
schiedliche Auffassungen der Vertragspartner hinsichtlich des geeignetsten Bieters, die auf vertragliche Regelungsdefizite zurückgeführt wurden.

\subsubsection{Die Feststellung und Bewertung von Nachtragsleistungen}

Mit 78\% sieht die Mehrheit der Experten die Feststellung und Bewertung von Nachtragsleistungen als weitestgehend unkritisch an. Als entscheidender Erfolgsfaktor hierfür wurde die transparente Ermittlung und die exakte Dokumentation der GMPPreisermittlungsgrundlagen genannt. Dadurch kann bereits ein erhebliches Konfliktpotenzial während der Preconstruction Phase eliminiert werden. Dies bestätigt auch die Aussage mehrerer Experten, dass ,bei frühzeitiger Einbindung des CM und gemeinsamer Bau-Soll-Definition“ i.d.R. keine Nachträge aus Vertragslücken entstehen.

Von mehreren Befragten wurden die beiderseitige Fairness und die Sensibilität für die Position des Projektpartners als Voraussetzungen genannt, um einen reibungsarmen Ablauf im Bereich des Nachtragswesens zu erreichen. Hierunter wird verstanden, dass der CM nur bei nachträglichen Eingriffen des AG in die Planung Nachtragsforderungen geltend macht und der AG im Gegenzug eine zeitnahe und objektive Prüfung vornimmt. Der Partnerschaftsgedanke muss folglich auch an dieser Stelle ,aktiv gelebt werden“.

$\mathrm{Zu}$ konkreten Problemen in den Projekten kam es im Wesentlichen dann, wenn von den v. g. Prämissen abgewichen wurde. Mehrfach wurde angeführt, dass Nachtragsstreitigkeiten vermehrt dann auftreten, wenn durch die späte Einbindung des CM kein einheitliches Vertragsverständnis entwickelt werden konnte. Einige Experten berichteten im gleichen Kontext, dass Probleme sehr häufig bei preisbasierter, funktionaler GU-Vergabe auftreten.

In einigen Projekten ergaben sich Differenzen zwischen den Projektpartnern, da die Grundlagen des GMP nicht hinreichend dokumentiert wurden. Weiteres Konfliktpotenzial war bei mangelnder Transparenz festzustellen. Der kausale Zusammenhang zwischen mangelnder Transparenz und Nachtragsstreitigkeiten wird auch durch die Aussagen weiterer Experten bestätigt, welche im Bereich der i.d.R. pauschalierten Eigenleistung tendenziell das größte Konfliktpotenzial innerhalb des Nachtragswesens sehen.

\subsubsection{Die Überwachung der Leistungserbringung durch den AN}

Die überwiegende Mehrheit von $89 \%$ der Befragten sieht die Überwachung der Leistungserbringung durch den AN als weitestgehend unkritisch an. Der Vorteil beim CM at risk liegt demnach in der Transparenz und Durchgängigkeit, da der AG direkt in die Qualitätsüberwachung der Nachunternehmer eingebunden werden kann. Insbesondere die Vereinbarung gemeinsamer Sachverständiger hat sich hierbei bewährt, da dies zu einer maximalen Akzeptanz und „gleichgerichtetem Handeln“ der Vertragspartner, zu einer schlanken Projektstruktur und zu einer Minimierung der beiderseitigen Kosten beiträgt.

Unabhängig davon haben sich aber auch Konstellationen bewährt, wo die Überwachung der Leistungserbringung durch den Architekten, die AG-Bauabteilung oder 


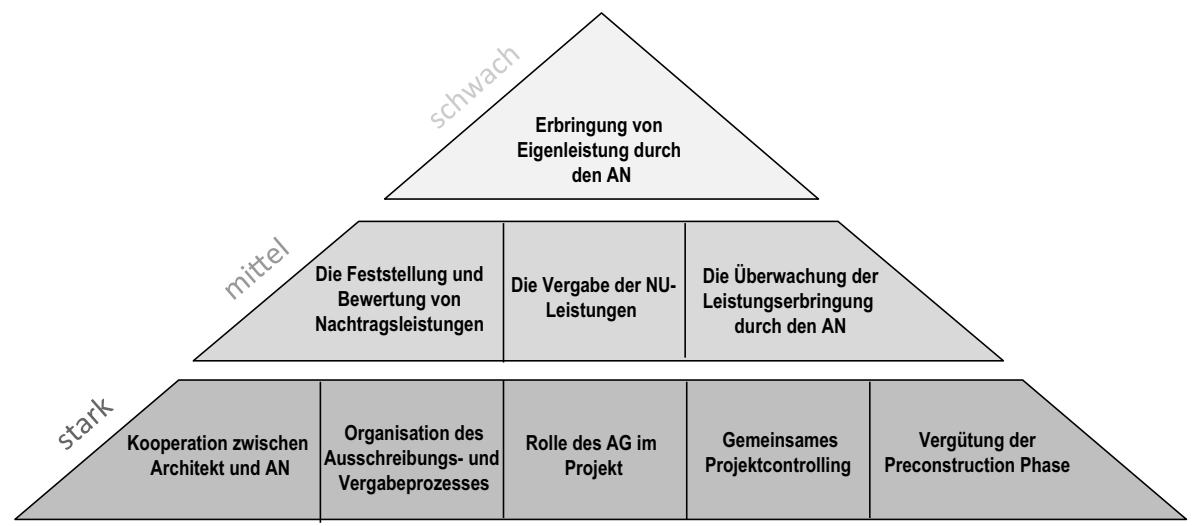

Abb. 11 Signifikanz der organisatorischen Handlungsfelder

eine externe Projektsteuerung erfolgte. Diese Situation führte jedoch in wenigen Projekten auch zu Konflikten zwischen den Vertragspartnern, was überwiegend auf die dadurch geschaffene Parallelorganisation zurückgeführt wurde.

Die Ergebnisse der durchgeführten Expertenbefragung zeigen, dass die verschiedenen organisatorischen Handlungsfelder des AG eine stark unterschiedliche Signifikanz in Bezug auf den Erfolg der Wertschöpfungspartnerschaft aufweisen (vgl. Abb. 11). Insgesamt wurden fünf kritische Erfolgsfaktoren identifiziert, denen bei der institutionellen Ausgestaltung der Wertschöpfungspartnerschaft eine erhöhte Aufmerksamkeit geschenkt werden muss.

Die im Rahmen der Expertenbefragung offengelegten Kausalzusammenhänge innerhalb der einzelnen Handlungsfelder dienen hierbei als Handlungsleitfaden, helfen Ineffizienzen $\mathrm{zu}$ verringern und unterstützen damit die strategische Ausgestaltung von $\mathrm{CM}$ at risk als eine effiziente Wertschöpfungspartnerschaft.

\subsubsection{Weitere Erfolgsfaktoren beim CM at risk}

Im Anschluss an die Fragen zu den Problemfeldern und Erfolgsfaktoren in den vordefinierten Handlungsbereichen der Projektorganisation wurde den Experten die Möglichkeit gegeben, eigene Parameter zu benennen, die sie für eine erfolgreiche Abwicklung von CM at risk als wichtig erachten. Die Antworten bewegten sich nahezu durchgängig im Bereich von Soft Skills, über welche beide Projektpartner zur erfolgreichen Realisierung von Projekten mit $\mathrm{CM}$ at risk verfügen müssen. Zudem wurden verschiedene Werkzeuge und Vorgehensweisen angeführt, welche die Herausbildung einer funktionierenden Wertschöpfungspartnerschaft unterstützen. Weitere organisatorische und vertragliche Parameter der Projektorganisation wurden im Rahmen der offenen Fragestellung von den Experten nicht genannt. Dieser Umstand lässt darauf schließen, dass die vom Verfasser vordefinierten Handlungsbereiche zutreffend und vollständig waren.

Die vielschichtigen Antworten der Experten im Rahmen der offenen Frage wurden im Zuge der Auswertung der Fragebögen inhaltlich geclustert. Unter Berücksichtigung der Anzahl der Nennungen ermöglicht diese Vorgehensweise Rückschlüs- 
se auf die Signifikanz des genannten Parameters innerhalb der Projektorganisation beim $\mathrm{CM}$ at risk.

85\% aller befragten Experten nannten die Partnering- bzw. Sozialkompetenz der handelnden Akteure seitens der beiden Vertragspartner als Grundvoraussetzung für eine erfolgreiche Projektrealisierung. Dieser Wert ist als Antwort auf eine offen gestellte Frage als sehr hoch anzusehen und ist damit als ein starkes Indiz für die außerordentliche Bedeutung dieses Parameters für den Erfolg der Wertschöpfungspartnerschaft anzusehen.

Als wichtige Voraussetzung für eine erfolgreiche Projektrealisierung sahen 59\% der Experten eine intensive Kommunikation zwischen den Projektpartnern an. Auch dieser Prozentsatz ist als hoher Wert in Beantwortung einer offenen Fragestellung anzusehen. Um die Kommunikation unter den Projektbeteiligten zu fördern, haben sich vor allem Instrumentarien bewährt, die möglichst kurze Kommunikationswege schaffen.

Auftraggeberseitig ist nach Auffassung von 35\% der Experten vor allem eine hinreichende Entscheidungskompetenz der wesentlichen Projektbeteiligten nötig. Dies erfordert kurze Entscheidungswege seitens des Bauherrn. Im Umkehrschluss bedeutet das, dass CM at risk als Projektorganisationsform bei komplexen Strukturen beim Einkauf von Bauleistungen, wie dies bei öffentlichen AG sowie bei Großkonzernen sehr oft der Fall ist, tendenziell ungeeignet ist.

Von 34\% der Befragten wurde eine hohe Dienstleistungskompetenz seitens des $\mathrm{CM}$ als unabdingbare Voraussetzung für den Erfolg der Projektabwicklung angesehen. Auch hierbei handelt es sich in Anbetracht der offen gestellten Frage um einen vergleichsweise hohen Wert, der eine entsprechend große Bedeutung dieses Parameters signalisiert.

\section{Diskussion der Ergebnisse}

Die institutionelle Ausgestaltung von CM at risk bzw. daran angelehnter Abwicklungsmodelle gestaltete sich in den untersuchten Projekten insgesamt sehr heterogen. Sie unterscheidet sich teilweise sehr deutlich vom erfolgreich praktizierten und weit verbreiteten $\mathrm{CM}$ at risk im angloamerikanischen Raum. Wendet man die Abgrenzungssystematik nach Kenig (2011) konsequent an, so handelt es sich bei der Mehrheit der in Deutschland realisierten Projekte aufgrund ihrer institutionellen Ausgestaltung nicht um CM at risk in definitorischem Sinne.

Darüber hinaus war bei vielen Projekten festzustellen, dass die ablauforganisatorische bzw. vertragliche Ausgestaltung einer effizienten Nutzung der Wertschöpfungspartnerschaft häufig diametral entgegenstehen. Die vielfach ungeeignete Ausgestaltung der Wertschöpfungspartnerschaft ist gleichzeitig ein starkes Indiz für ein mangelndes Verständnis seitens der ökonomischen Akteure in Bezug auf die grundlegenden Kausalzusammenhänge zwischen den verschiedenen Gestaltungsparametern und dem Verhalten der Projektbeteiligten.

In Anbetracht der erheblichen Heterogenität der untersuchten Wertschöpfungspartnerschaften sowie der vielfach ungeeigneten institutionellen Ausgestaltung überrascht es daher nicht, dass diese bislang mit stark unterschiedlichem Erfolg eingesetzt 
werden. Das Effizienzsteigerungspotenzial dieser Projektorganisationsform kann folgerichtig nur unzureichend ausgeschöpft werden. Die theoretischen Vorüberlegungen $\mathrm{zu}$ den Konsequenzen einer ungeeigneten Ausgestaltung von Organisationsaufbau und Verfahrensablauf in Bezug auf die Kooperation der Wertschöpfungspartner wurden durch die Expertenbefragung weitestgehend bestätigt.

Die Studie hat gezeigt, wie sich durch eine geeignete institutionelle Ausgestaltung in den verschiedenen organisatorischen Handlungsfeldern Rahmenbedingungen schaffen lassen, die ein effizientes Zusammenwirken der Wertschöpfungspartner gewährleisten. Dadurch wird die Voraussetzung geschaffen, dass das Effizienzsteigerungspotenzial dieser Beschaffungsvariante bestmöglich ausgeschöpft werden kann. Speziell die Ausgestaltung der als kritische Erfolgsfaktoren identifizierten Parameter der Projektorganisation sind dabei von erhöhter Bedeutung. Zusätzlich zu einer geeigneten Projektaufbau- und Ablauforganisation setzt ein erfolgreiches CM at risk auch verschiedene Soft Skills seitens der Projektpartner voraus. Diese sind maßgebliche Eignungsvoraussetzungen der Wertschöpfungspartner und zählen damit ebenfalls zu den kritischen Erfolgsfaktoren.

Die Kenntnis der durch die empirische Studie erschlossenen Kausalzusammenhänge innerhalb der einzelnen Handlungsfelder in Kombination mit der Identifikation der kritischen Erfolgsfaktoren ermöglicht die strategische Gestaltung und Steuerung einer effizienten Wertschöpfungspartnerschaft.

Die gewonnenen Erkenntnisse bilden die Basis für die Entwicklung eines theoretischen Organisationsmodells, welches zu einer Standardisierung und damit zur Professionalisierung der ökonomischen Akteure in der Bau- und Immobilienwirtschaft beiträgt.

Open Access Dieser Artikel wird unter der Creative Commons Namensnennung 4.0 International Lizenz (http://creativecommons.org/licenses/by/4.0/deed.de) veröffentlicht, welche die Nutzung, Vervielfältigung, Bearbeitung, Verbreitung und Wiedergabe in jeglichem Medium und Format erlaubt, sofern Sie den/die ursprünglichen Autor(en) und die Quelle ordnungsgemäß nennen, einen Link zur Creative Commons Lizenz beifügen und angeben, ob Änderungen vorgenommen wurden.

\section{Literatur}

Acker W (2006) Außergerichtliche Streitschlichtung unter Einbeziehung angloamerikanischer Modelle. In: Innovative Abwicklungsformen für Bauprojekte: Partnering und PPP. Tagungsband. Schriftenreihe Bauwirtschaft. kassel university press, Kassel

Bennett J, Jayes S (1995) Trusting the team - The best practice guide to Partnering in construction. University of Reading, Reading

Bogner A, Littig B, Menz W (2014) Interviews mit Experten. Springer, Wiesbaden

Bücker M (2005) Construction Management, Untersuchungen zur Anwendbarkeit der US-amerikanischen Projektabwicklungsform Construction Management at Risk in Deutschland. Shaker Verlag, Aachen (Diss.)

Cunningham $\mathrm{G}$ (2005) Commissioning large public projects using construction manager at risk (CM@ risk). American Institute of Architects (AIA), Washington, DC

Eschenbruch K, Racky P (Hrsg) (2008) Partnering in der Bau- und Immobilienwirtschaft. Kohlhammer, Stuttgart

Gläser J, Laudel G (2010) Experteninterviews und qualitative Inhaltsanalyse, 4. Aufl. VS, Wiesbaden

Gralla M (2001) Garantierter Maximalpreis - GMP-Partnering Modelle - Ein neuer und innovativer Ansatz für die Baupraxis. B. G. Teubner, Stuttgart

Gransberg DD, Shane JS (2010) Construction manager-at-risk project delivery for highway programs. Transportation Research Board of the national academies, Washington D.C.

Haltenhoff CE (1999) The CM contracting system: Fundamentals and practices. Prentice Hall, New Jersey 
Heidemann A (2010) Kooperative Projektabwicklung im Bauwesen unter der Berücksichtigung von LeanPrinzipien - Entwicklung eines Lean-Projektabwicklungssystems. Karlsruher Institut für Technologie, Karlsruhe (Diss.)

Kenig ME (2011) Project delivery systems for construction, 3. Aufl. AGC of America, Arlington

Konchar M (1997) A comparison of US project delivery systems. Pennsylvania State University, Old Main

Loskant D (2010) Der wettbewerbliche Dialog im Kontext der Partnerschaftsmodelle. Shaker Verlag, Aachen (Diss.)

Mathoi T (2006) Maximalpreismethode. Books on Demand, Norderstedt

Meyer K, Pfnür A (2015) Wertschöpfungspartnerschaften in der Immobilienprojektentwicklung. Z Immobilienökonomie 1(1):59-80. https://doi.org/10.1365/s41056-015-0003-9

Mütze M, Abel M, Senff T (2009) Immobilieninvestitionen - Die Rückkehr der Vernunft. Rudolf Haufe, München

Ohler FP, Wolff R, Richter T (2012) Beteiligte des Bau- und Planervertrages. In: Messerschmidt B, Voit W (Hrsg) Privates Baurecht, 2. Aufl. C.H. Beck, München

Pfnür A (2011) Modernes Immobilienmanagement, 3. Aufl. Springer, Berlin

Roland Berger Strategy Consultants (2008) Partnerschaftsmodelle als Erfolgsfaktor in der Bauindustrie. www.rolandberger.com

Schlabach C (2013) Untersuchungen zum Transfer der australischen Projektorganisationsform Project Alliancing auf den deutschen Baumarkt. kassel university press, Kassel (Diss.)

Schmolke D (2008) Innovation durch Partnering im Deutschen Schlüsselfertigbau. Wuppertal (Diss.)

Tautschnig A, Hulka G (2002) Die besondere Eignung des GMP-Modells für „Fast-Track“-Projekte im Hochbau. Bauingenieur 77(10):484-492 